\title{
Interactive comment on "Linking sea ice deformation to ice thickness redistribution using high-resolution satellite and airborne observations" by Luisa von Albedyll et al.
}

\section{Anonymous Referee \#1}

Received and published: 21 November 2020

General comments:

This manuscript deals with an unusual ice deformation event that occurred off the northeastern coast of Greenland in early 2018. The authors make use of SAR data and ice thickness derived from an airborne survey to understand the ice deformation history within a polynya that opened rapidly and refroze over the course of one month. The authors address the contributions of thermodynamic and dynamic processes to the geometric shape of the ice thickness distribution within the polynya. The data and analysis presented are of interest to the community, and the study may warrant publication after revisions to address the concerns listed below. I found the manuscript text 
itself to be extremely long, and shortening some sections could really improve things. Thorough copyediting throughout is also required.

Specific comments:

Polynya:

What type of polynya is this (latent or sensible heat), and how did it form? I think a brief discussion about the geophysical characteristics of this polynya, and specific details of its formation, are warranted in the introduction/background information. On L63 the authors refer to an "unusually strong and persistent atmospheric pattern" that drove formation of the polynya. But I think this statement is too vague to understand the forcing mechanism/s. What type of atmospheric pattern? Was the polynya formation wind driven, temperature driven, or both? They say that the atmospheric pattern reversed the "normally coastward direction of the large-scale ice drift close to northeast Greenland". Is there a reference (publication or data set/analysis) to corroborate this statement about the typical ice drift direction? Is ice drift normally coastal in this area in winter, or is it actually parallel to the coastline? Knowledge of the processes driving daily thickness change is also needed to interpret the data shown in Fig 4. If the polynya opened as a result of a wind-driven event, rather than a local heat event, then the thickness change that occurred at the beginning of the time series was dynamic (i.e. thicker MYI replaced by open water). This point does not appear to be fully captured.

Ice type:

The survey area (shown in Figure 1 and discussed in the text, L98) is described as a region of first year ice (FYI), but I don't think this is accurate. (1) The airborne survey was conducted over both FYI and multi-year ice (MYI) as shown in 1(a) - AEM tracks shown by both coloured dots, and thick white lines, traverse areas of high and low SAR backscatter indicating a mixture of ice types. (2) There seem to be many MYI floes within the polynya itself that were either advected into the polynya (as the authors

Printer-friendly version

Discussion paper

2 
assume) or that remained in the polynya after the temporally abrupt opening event. I'm curious to know how the thickness of these thicker MYI floes within the polynya (that were traversed by the airborne survey) were excluded (L120) from the ice thickness distributions shown in $1(\mathrm{~d})$ and $3(\mathrm{a})$. Since there is MYI within the FYI area (Fig 1a), and new ice forming in leads within the MYI region (Fig 3b, L332) a qualifier regarding ice type should be included throughout. It would be more accurate to describe a region as having a "dominant" ice type rather than only one ice type.

SAR analysis:

The description of how the SAR data are analyzed is vague with no specific details about how ice type was derived, nor how ice parcels were identified for drift tracking, or the derivation of deformation (divergence and shear rates). This means the analysis presented is irreproducible, and does not lend itself to the future work proposed on L650-651. For example, rather than quantitively referring to the SAR data presented in Figure 1 (a) and (c) as "light" and "dark to distinguish areas in the study region I recommend something more quantitative. Consider providing threshold values, or ranges, of backscatter (in $\mathrm{dB}$ ) that can be used alongside a colour scale with appropriate units, to define ice type. How were the data used to define the "rim" of the polynya? i.e. was a specific back-scatter threshold used here? If so, please include the details of this in the text. Similarly, how was the sheer zone within the polynya identified and defined? How were regions of fast ice defined? (Fig 1a) Was SAR backscatter utilized to define these zones?

Modal ice thickness:

I found that there was an error of $0.05-0.1 \mathrm{~m}$ throughout the study in the reporting of modal ice thickness. If the bin width of the thickness distributions is $20 \mathrm{~cm}$, as shown in the figures, then the modes reported in the text are not consistent with the data shown in the figures. Isn't the mode of the thickness distribution shown in Fig $1 \mathrm{~d} 1.1 \mathrm{~m}+/-0.1$ $\mathrm{m}$ (not $0.95 \mathrm{~m}$ as written on L70, L306-307)? Likewise, the mode of the level ice shown

Printer-friendly version

Discussion paper 
in Fig 3a is $0.9 \mathrm{~m}$, (not $0.95 \mathrm{~m}$, as stated on L319). The primary mode shown in Fig 3b appears to be $0.1 \mathrm{~m}+/-0.1 \mathrm{~m}$, and the secondary mode is $1.9 \mathrm{~m}+/-0.1 \mathrm{~m}$ (and not 2.0 $\mathrm{m}$, as stated on L335).

\section{Data:}

The majority of the data used in this study lack citations, or are not public, meaning the analysis is not reproducible. For example, the citation for ice drift data (a web URL to a generic data page) is not sufficient, and is not indicative of the particular data set used. Similarly for the IceBridge data, no product is referenced. Are the AEM data publicly available?

\section{Language:}

Overall, the manuscript needs to be revised to improve English usage and grammar, since there are many functional mistakes in the formulation of key sentences. Substantial copyediting is required to aid understanding. I've suggested some specific edits within the technical list below.

Technical corrections:

L11: Change "by drift tracking along Lagrangian backward trajectories" -> by tracking ice drift along reverse Lagrangian trajectories to go back in time

L16-17: Change "The computed ice thickness distribution resembles main characteristics like mode, e-folding, and width of the observed distribution" $\rightarrow$ The computed ice thickness distribution resembles the main characteristics of the observed distribution including mode, e-folding, and width.

L17: clarify what is mean by width, do you mean "full width half maximum"?

L19-20: Change "The similar first- and multi-year ice mean thicknesses elude to the large amount of deformation experienced by the closing polynya" $\rightarrow$ the similarity between the mean thickness of first- and multi-year ice suggests the scale of deformation 
that occurred within the closing polynya.

L30-32: the use of "mean peak depth" to describe keel depths does not sound right. I suggest rewording this using a term such as modal depth. Also, I appreciate that there's a lot of statistics presented in Strub-Klein and Sudom (2012) and so I would suggest here that you clarify which observational statistics you are quoting. I believe they relate to "maximum" sail heights and keel depths. Here you might also refer to Duncan et al. (2020) which updates and expands upon the Strub-Klein and Sudom (2012) work, and found that the average maximum sail height was $2.01 \mathrm{~m}$ for $>17,000$ ridges formed in $\mathrm{FYI}$.

L45-46: Provide a suitable reference to support this statement.

L52: Remove "so far" since it is not needed in this sentence. Also change "changed" $\rightarrow$ changes in

L65: the text refers to the date of the maximum extent of the polynya as February 25th, but the figure (Fig 1c) shows a SAR image from March 1st.

L66: change "unprecedented" $\rightarrow>$ unusually

L68: revise "coastward directed winds" with a vector direction

L69-73: Is this a statement of work described elsewhere (i.e., previously published) or a summary of the work we are about to read about in this manuscript? If the former, provide a suitable reference; if the latter, move this statement from the introduction to the conclusion section.

L96: for completion I suggest you include the year to any dates provided within the text, in addition to month and day.

L105: by "peak" do you mean modal, or maximum?

L109-111: What Operation IceBridge data product for snow thickness is used here?

What is the uncertainty associated with an airborne snow thickness observation of 4 
$\mathrm{cm}$ ?

L109-111: How much does uncertainty in snow thickness contribute to errors in the attribution of thermodynamic processes to the overall ITD?

L113-115: how does this assumption impact the uncertainty associated with the AEM thickness estimates, relative to that stated on L105?

L118-119: unfortunately this is not possible for the reader since there is no colour scale provided with the SAR data shown in Fig 1, nor is it clear what the units are.

L121-122, L149-151: I'm curious to understand what is meant by "visual interpretation of the SAR backscatter signature". Is this done using linear pixel greyness values, and therefore subjective, or by applying thresholds to the SAR data in $\mathrm{dB}$ ? How are MYI floes defined and excluded?

L125: according to the figures, the bin width is $20 \mathrm{~cm}$.

L166: can you explain what is meant by "radar intensity"?

L127: change "Large e-folding and FWHM" $\rightarrow$ to large values of e-folding and FWHM

L142: can you briefly describe what is meant by "two-category, zero-layer thermodynamics"?

L153: change "adding up" $\rightarrow$ summing

L153-154: does 't' represent thickness, or time?

L159-160: Snow depth on thin ice has a large control on thermodynamic ice growth. How was thermodynamic growth impacted by snow thickness changes (and/or snow redistribution) over 30 days? Does imprecise knowledge of this impact the conclusions drawn?

L174: Provide an example of the derived ice drift data so that the reader may evaluate the results for reasonableness 
L182: what are the variables $u$ and $v$ in equation 2 ?

L171: Provide an example of the derived ice deformation data so that the reader may evaluate the results for reasonableness

L191: Did the authors compute uncertainty in the derived divergence, shear and deformation fields?

L195: "In order to coincide with the surveyed ice" - this is quite awkward, consider rewording.

L200: what is the delta time between the airborne survey and image acquisition?

L202: Change "at the time step before" $\rightarrow$ at the previous time step

L210: how is the reliability of the tracking algorithm quantified?

L212-213: another very awkward sentence that is hard to follow - consider rewording

L220-224: Can you show this assessment?

L225: change "sums up" $\rightarrow>$ accumulates

L291, L293: is there a reason why the number of combinations and iterations are reported to three significant figures? Are combinations and iterations the same thing?

L306 and L332: how is open water fraction computed?

L307: "with most of the ice" - state the \% here

L310" Change "evenly" -> even, or is there a word missing here?

L310: is uniform thermodynamic growth "expected" over such a large area? I think it is assumed (but not necessarily expected) since we do not know about snow distribution.

L312-313: "Deformation has led to the presence of a long tail of the distribution up to $20 \mathrm{~m}$ thickness" - But the scale in in Fig 3a only shows data to $8 \mathrm{~m}$. What \% of samples in the tail span $8 \mathrm{~m}$ to $20 \mathrm{~m}$ ? Consider adding AEM profile data here to substantiate 
this statement (similar to the data shown in Fig 6).

L320: Are the modes identical? Looking at Fig 3a, it appears the mode of the level ice thickness distribution is less than that of the 'complete' ice thickness distribution.

L335: Isn't the modal thickness $1.95 \mathrm{~m}$ (Fig 3b)?

L560: Did the authors consider the ice thickness distribution from CryoSat-2 for this region so as to substantiate their statement?

L564: double "of"

L586: change "we suggest to couple the SAR deformation retrievals with. .." -> we suggest coupling deformation statistics retrieved from SAR analysis with ....

L630, 632: change "coverage" $\rightarrow$ cover

L645-655: this sentence is quite hard to follow - consider rewording

Figure 1:

In 1(a) the drift trajectories (thin white lines with arrows) are not defined in the legend. What is the reasoning behind the uneven increments used in the color scale for ice thickness? Why, for example, is the majority of ice (according to figure 1d) combined, and represented by only one colour increment (light green) while thicker ice is divided into four increments ranging between 0.15 and $0.24 \mathrm{~m}$ in thickness? A scale bar for the blue arrows in 1 (b) is required, showing drift magnitude. Change "stippled" to "dashed" in the figure caption. Do the data in 1 (a) and (d) show ice thickness (as stated in the figure caption) or ice thickness + snow depth (as stated on L98-99)?

Figure 3:

Indicate in the axis labels for (a) and (b) whether you show ice thickness + snow depth or ice thickness only. From my reading of the text I think (a) is the distribution of ice thickness, but (b) is the distribution of ice + snow thickness. Is the "complete" thickness 
distribution shown in Fig 3a repetition of the data shown in Fig 1d? If so, remove one of these duplicate figures.

Interactive comment on The Cryosphere Discuss., https://doi.org/10.5194/tc-2020-303, 2020. 